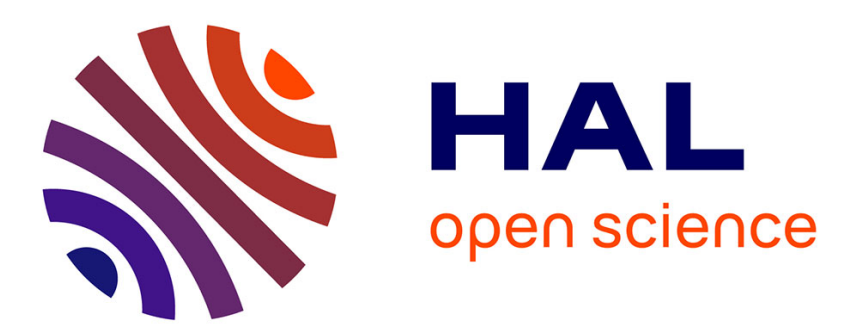

\title{
Obtention of Giant Unilamellar Hybrid Vesicles by Electroformation and Measurement of their Mechanical Properties by Micropipette Aspiration
}

\author{
Emmanuel Ibarboure, Martin Fauquignon, Jean-François Le Meins
}

\section{- To cite this version:}

Emmanuel Ibarboure, Martin Fauquignon, Jean-François Le Meins. Obtention of Giant Unilamellar Hybrid Vesicles by Electroformation and Measurement of their Mechanical Properties by Micropipette Aspiration. Journal of visualized experiments: JoVE, 2020, 155, pp.e60199. 10.3791/60199 . hal02446686

\section{HAL Id: hal-02446686 \\ https://hal.science/hal-02446686}

Submitted on 9 Apr 2020

HAL is a multi-disciplinary open access archive for the deposit and dissemination of scientific research documents, whether they are published or not. The documents may come from teaching and research institutions in France or abroad, or from public or private research centers.
L'archive ouverte pluridisciplinaire HAL, est destinée au dépôt et à la diffusion de documents scientifiques de niveau recherche, publiés ou non, émanant des établissements d'enseignement et de recherche français ou étrangers, des laboratoires publics ou privés. 


\title{
OBTENTION OF GIANT UNILAMELLAR HYBRID VESICLES BY ELECTROFORMATION AND MEASUREMENT OF THEIR MECHANICAL PROPERTIES BY MICROPIPETTE ASPIRATION TECHNIQUE
}

\author{
AUTHORS AND AFFILIATIONS: \\ Emmanuel Ibarboure*, Martin Fauquignon, Jean-François Le Meins* \\ Univ. Bordeaux, CNRS, Bordeaux INP, LCPO, UMR 5629, F-33600, Pessac, France \\ martin.fauquignon@enscbp.fr
}

Corresponding authors

ibarbour@enscbp.fr

lemeins@enscbp.fr

KEYWORDS:

Giant Unilamellar Vesicles

Micropipette

Copolymer

Lipid

Hybrid Polymer/Lipid vesicle

Membrane properties

Area compressibility modulus

Bending modulus

Electroformation

Bilayer

\section{SUMMARY:}

The goal of the protocol is to show how to obtain reliable measurement of membrane mechanical properties on Giant vesicles by micropipette aspiration technique.

\footnotetext{
ABSTRACT:

Giant vesicles obtained from phospholipids and copolymers can be exploited in different applications such as: controlled and targeted drug delivery, biomolecular recognition within biosensors for diagnosis, functional membranes for artificial cells, development of bioinspired micro-/nano-reactors. In all of these applications, the characterization of their membrane properties is of fundamental importance. Among existing characterization techniques, micropipette aspiration, pioneered by E. Evans, allows the measurement of mechanical properties of the membrane such as area compressibility modulus, bending modulus and lysis stress and strain. Here we present all the methodologies and detailed procedures to obtain giant vesicles from thin film of lipid or copolymer (or both), manufacturing and surface treatment of micropipettes and aspiration procedure leading to the measurement of all parameters previously mentioned.
} 


\section{INTRODUCTION:}

Giant vesicles obtained from phospholipids (liposomes) has been widely used since the 1970's mostly as basic cell's membrane model. ${ }^{1}$ In the late 1990 's, vesicular morphologies obtained from the self-assembly of copolymers, named polymersomes in reference to their lipid analogs, ${ }^{2,3}$ rapidly appeared as an interesting alternative to liposomes which possess weak mechanical stability and poor modular chemical functionality. However, their cell biomimetic character is rather limited compared to liposomes since the latter are composed of phospholipids, the main component of the cell membrane. Furthermore, their low membrane permeability can be an issue in some applications like drug delivery where controlled diffusion of species through the membrane is required. These last years, the association of phospholipids with block copolymers to design hybrid polymer-lipid vesicles and membranes has been the subject of an increasing number of studies. ${ }^{4,5}$ The main idea is to design entities that synergistically combine the benefits of each component (bio-functionality and permeability of lipid bilayers, mechanical stability and chemical versatility of polymer membranes), which can be exploited in different applications such as controlled and targeted drug delivery, biomolecular recognition within biosensors for diagnosis, functional membranes for artificial cells, development of bio-inspired micro-/nano-reactors.

Nowadays, different scientific communities (biochemists, chemists, biophysicists, physicochemists, biologists) present an increasing interest regarding the development of more advanced cell's membrane model. Here our goal is to present, as detailed as possible, existing methodologies (electroformation, micropipette aspiration) to obtain and characterize the mechanical properties of Giant vesicles and the recent "advanced" cell membrane models that are Hybrid Polymer Lipid Giant Vesicles. ${ }^{4,5}$

The purpose of these methods is to obtain reliable measurement of the area compressibility and bending moduli of the membrane as well as their lysis stress and strain. One of the most common technique existing to measure bending rigidity of a Giant vesicle is the fluctuation analysis $^{6,7}$, based on direct video microscopy observation but it requires large visible membrane fluctuation, not systematically obtained on thick membranes (e.g. polymersomes). Area compressibility modulus can be experimentally determined using Langmuir Blodgett technique but most often on monolayer. ${ }^{8}$ Micropipette aspiration technique allows the measurement, in one experiment of both moduli on bilayer forming GUV.

The following method is appropriate for all amphiphilic molecules or macromolecules able to form bilayers and consequently vesicles by electroformation. This requires a fluid character of the bilayer at the temperature of electroformation.

\section{PROTOCOL:}

\section{Protocol for fabricating micropipettes:}

In our experiments, we need to develop capillaries with an inner diameter ranging from 6 to 12 $\mu \mathrm{m}$ and a taper length around 3-4 $\mathrm{mm}$. A detailed method of manufacturing micropipette is described in the following:

1.1. Place the borosilicate glass capillary in the drawbar of the puller and fix one of the ends by 
tightening the knob.

1.2. Carefully slide glass through the holes at the side of the heater chamber.

1.3. Tighten down clamping knob at the other end.

1.4. Control the size of the tip and the taper length to achieve the desired specifications. For that, optimize technical parameters such as heating temperature (control the power of current supplied to the filament), pull (control the force of the pull), velocity (this value is determined by the viscosity of the glass capillary), delay and pressure. Here is an example of a program used:

Heat: $550^{\circ} \mathrm{C}$

Pull: 10 (Range of the machine: 0-255 in arbitrary units)

Velocity: 30 (Range: 0-255 in arbitrary units)

Delay: 1 (Range: 0-255 in arbitrary units)

Pressure: 500 (Range: 0-999 in arbitrary units)

1.5. Click on "PULL" to execute the events defined by the program. The capillary is then separated into two micropipettes whose dimensions have to be adjusted using a microforge.

1.6. Insert the micropipette into the metal pipette holder of the microforge (see Figure 1). By using 10x objective, adjust the microscope stage and the pipette manipulator (vertical and horizontal movement) until the pipette tip is close to the glass bead surface.

1.7. Press the foot switch to melt the glass bead. Lower the tip and put it in contact with the molten glass bead. Molten glass will flow into the pipette by capillarity. Wait a few seconds until the level of the molten glass reaches a certain height as shown in the insert of the Figure 1.

1.8. Stop the heating by removing the pressure on the foot switch, and quickly pull the tip away using the vertical pipette manipulator to cause a sharp break. Repeat steps 1.7 and 1.8 until the desired diameter is obtained (6 to 12 microns).

NOTE: To improve the accuracy of the diameter measurement, during the last step, use a $32 \mathrm{X}$ objective equipped with a reticle.

\section{Protocol for Coating pipette tips with BSA (Bovine Serum Albumin)}

2.1. To prepare a $0.1 \mathrm{M}$ solution of glucose containing $1 \mathrm{wt}$. \% BSA in pure water.

2.1.1. Basically, weigh $180 \mathrm{mg}$ of glucose powder, put it in a $15 \mathrm{~mL}$ polypropylene conical tube and complete with $10 \mathrm{~mL}$ of pure water.

2.1.2. Add $0.1 \mathrm{~g}$ of BSA powder and shake gently using a test tube rotatory mixer until complete dissolution (approximately 4 hours).

2.2. Take the solution with a $10 \mathrm{~mL}$ disposable luer syringe fitted with a needle. Once filled, remove the needle and install a $0.22 \mu \mathrm{m}$ acetate cellulose filter. Fill several polypropylene micro-centrifuge tubes $(1.5 \mathrm{~mL})$ that will be used to immerse the tip.

2.3. Place the capillaries vertically into holders. Lower the holder and immerse the tip into the glucose / BSA solution overnight. The solution should rise about $1 \mathrm{~cm}$ high by capillarity.

2.4. Remove the pipette tip from the glucose/BSA solution. Prepare $5 \mathrm{ml}$ of $0.1 \mathrm{M}$ glucose 
solution (dilute $90 \mathrm{mg}$ glucose powder in $5 \mathrm{ml}$ of pure water) and filter through $0.22 \mu \mathrm{m}$ acetate cellulose filter.

2.5. Fill the pipette with the glucose solution by using a $500 \mu$ l glass syringe equipped with a flexible fused silica capillary. Then, remove all the glucose solution by sucking it back and discard it (Figure 2). Repeat this step several times to remove the unbounded BSA.

\section{Formation of GUVs and GHUVs by electroformation}

Electroformation is a commonly used technic developed by Angelova and col. ${ }^{9}$ to form GUVs from amphiphilic molecules such as phospholipids or copolymers. The alternating potential difference applied across the electrodes stimulates the hydration and swelling process of the layers. The procedures to obtain an electroformation chamber, deposit a lipid or polymer film (or both for GHUV) and hydrate the film under alternative electric field are described in the following. The procedure to collect the GUV obtained is also described.

\subsection{Amphiphile, sucrose and glucose solutions preparation}

3.1.1 Prepare an amphiphile solution at a concentration of $1 \mathrm{mg} \cdot \mathrm{ml}^{-1} .10 \mathrm{mg}$ of amphiphile is weighted and dissolved in $10 \mathrm{ml}$ of chloroform. The solution is kept in sealed vials to avoid solvent evaporation.

3.1.2. Prepare a stock solution of 1,2-dioleoyl-sn-glycero-3-phosphoethanolamine-N-(lissamine rhodamine $B$ sulfonyl) (DOPE-Rhod) at $1 \mathrm{mg} \cdot \mathrm{ml}^{-1}$ in chloroform.

3.1.3 Add $10 \mu \mathrm{L}$ of fluorescent lipid solution to the amphiphile solution. Keep the solutions in sealed vials to avoid solvent evaporation.

3.1.4. Prepare sucrose and glucose solutions at a concentration of $0.1 \mathrm{~mol} . \mathrm{L}^{-1}$. Weight $342 \mathrm{mg}$ and $180 \mathrm{mg}$ of sucrose and glucose respectively and dissolve them in $10 \mathrm{ml}$ of pure water.

\subsection{Preparation of the electroformation chamber}

Different conductive materials can be used to make an electroformation device (e.g. platinum wires $^{10}$, stainless needles ${ }^{11}$ ). Here we describe how to construct a device made from glass plates coated with indium tin oxide (ITO). The electroformation chamber is composed of two ITO slides separated by an O-ring rubber spacer which has been cut on one side to create an aperture. The slides are connected to a voltage generator via two electric wires attached at the slide surface by adhesive tape. (Figure 3 and 4-A))

3.2.1 Clean the ITO slides with organic solvent (e.g. chloroform). Identify conductive surface using an ohmmeter.

3.2.2. Attach the electrical wires on the conductive side using adhesive tape.

3.2.3. Amphiphile solution deposition:

3.2.3.1 Dip a capillary in the solution until the level increases by capillarity and collect about $5 \mu l$ of the solution.

3.2.3.2 Put the capillary in contact with the center of the ITO glass plate and gently spread the solution. Wait 10 seconds to ensure the complete solvent evaporation. (Figure 4-A)

3.2.3.3 Repeat this procedure 3 times for each sides.

3.2.4. Put a layer of silicon-free grease on both sides of the opened O-ring spacer. Put it around 
the area of deposition. Place the conductive face of the second ITO glass plate on the top of the spacer.

3.2.5. Place the electroformation chamber under vacuum during 3 hours to remove any traces of organic solvent.

\subsection{Electroformation procedure}

3.3.1. Plug the electric wires to the generator.

3.3.2. Use the following settings for the generator:

Alternative sinusoidal tension

Frequency: $10 \mathrm{~Hz}$

Amplitude: $2 \mathrm{~V}_{\text {peak-to-peak }}$

NOTE: optimum voltage frequency and duration has to be found for each system

3.3.3. Ensure that the voltage is applied before injection of the solution in the chamber.

3.3.4. Inject $1 \mathrm{ml}$ of solution using a syringe with $0.8 \mathrm{~mm}$ inner diameter needle to fill the chamber. Remove eventual bubbles.

3.3.5. Let the chamber under the applied voltage/frequency during $75 \mathrm{~min}$. (Figure 4-B)

\subsection{GUVs harvest}

\subsubsection{Switch off the generator.}

3.4.2. Using $1 \mathrm{ml}$ syringe with $0.8 \mathrm{~mm}$ inner diameter needle, suck a small part of the solution in order to produce an air bubble inside the chamber. Slightly tilt the chamber in order to make this bubble move inside the chamber. This can help the GUVs to detach from the ITO surface (Figure 4-C).

3.4.3. Suck all the solution and transfer it into a $1 \mathrm{ml}$ plastic tube.

3.4.4. Remove the wires and clean the ITO slides with organic solvents (toluene then chloroform).

\section{Micromanipulation Set up}

Micropipette aspiration is a common technique to measure the mechanical properties of giant vesicles. The principle is to suck a single vesicle through a glass micropipette by applying a depression. The length of the tongue inside the pipette is measured as a function of the suction pressure.

The pipette coating with BSA, described previously, is essential to prevent or minimize any adhesion between liposomes or polymersomes membranes and the glass.

The protocol is illustrated below.

\subsection{Pipette and water filled reservoir connection:}

The water filled tank and the micrometer are fixed to a sliding plate. A digital counter with a micrometer head allows a vertical displacement of the device in a range of 0 to $2.5 \mathrm{~cm}$ and an 
accuracy of $1 \mu \mathrm{m}$. This set up can also slide along an aluminum optical rail of 1 meter in length. The reservoir and the capillary metal holder should be connected to each other by silicone tubing, as shown in Figure 5-A. The silicone tubing is identified as number 3 in this Figure.

4.1.1. Fill the tank with pure water. Connect a disposable $5 \mathrm{ml}$ syringe to the capillary metal holder by silicone tubing and aspirate to create a water flow from the reservoir to the holder. 4.1.2. Touch the tube slightly to eliminate air bubbles. Simultaneously, raise the water tank to create a positive pressure. The $5 \mathrm{ml}$ syringe is still attached to the holder.

4.1.3. After coating and cleaning steps described previously (see "Coating pipette tips with BSA" section), fill a capillary with glucose solution until a drop forms at the end. Remove the syringe tubing from the metal holder to create a slight water flow at its end.

4.1.4. Turn the capillary upside down and connect the glucose drop with the water flow from the holder. Fix the capillary and the holder by screwing them together.

\subsection{Position a pipette:}

NOTE: During this operation, the water tank is still positioned on the aluminium rail to maintain a positive pressure.

4.2.1. Take the homemade aluminum stage equipped with two glass slides (previously cleaned with ethanol) and glue them with vacuum grease on each side. Install it on the microscope stage and form a meniscus between the two slides by using a $1 \mathrm{ml}$ syringe containing $0.1 \mathrm{M}$ of glucose as shown in Figures 5-B and 5-C.

4.2.2. Place the pipette and its holder on the motor unit of the micromanipulator and tighten down clamping knob.

4.2.3. Use the control panel joystick in coarse mode to lower the micropipette near the glucose meniscus. Adjust the position of the tip to the center of the meniscus using the fine mode.

4.2.4. Hold the tip immersed in glucose for a few minutes to clean its outer and inner surface (as a positive pressure is maintained, the water flow will rinse the inner surface of the pipette to remove uncoated BSA)

4.2.5. Store the position of the tip on the micromanipulator keyboard and withdraw it from the meniscus.

4.2.6. Remove the glucose meniscus and replace it by a fresh one. Suck $2 \mu \mathrm{L}$ of GUVs in $0.1 \mathrm{M}$ sucrose by using a $20 \mu \mathrm{l}$ micropipette and put it in the fresh glucose meniscus. Observe in DIC mode (Differential interference contrast) the GUVs located at the bottom due to the difference of density between sucrose (mainly inside the GUVs) and glucose (mainly outside the GUVs).

4.2.7. When the vesicles are slightly deflated, reinsert the suction pipette and focus on the tip. The height of the water tank for which pressure is almost 0 has to be set. For that, take advantage of small vesicles or particles which are naturally present in solution and adjust the water tank height until the motion of these particles is stopped. This height will be considered as the origin $\mathrm{H}_{0}$.

4.2.8. At this point, surround the meniscus with mineral oil to prevent water evaporation, see Figure 5-D. 
NOTE: The room temperature should be controlled between $20-22^{\circ} \mathrm{C}$ by using air conditioning.

\subsection{Micropipette aspiration experiment:}

4.3.1. Lower the pipette tip (6-12 $\mu \mathrm{m}$ in diameter) and create a small suction $(-1 \mathrm{~cm})$ to aspirate a vesicle (15-30 $\mu \mathrm{m}$ in diameter). The membrane of the selected vesicle should slightly fluctuate, and must not present any visible defects (no bud or filament). (Figure 6)

4.3.2. Raise the pipette to a higher level to isolate the aspirated vesicle from the other vesicles, by using the micromanipulator and keep this position during the whole experiment.

4.3.3. Pre-stress the vesicle by lowering the water filled tank to approximately $-10 \mathrm{~cm}$, then decrease the pressure to return to its initial value $(-1 \mathrm{~cm})$. Repeat this step several times to remove membrane excess and small defects from the membrane.

4.3.4. From a height of $-0.5 \mathrm{~cm}$ defined by the position of the water tank, slowly decrease the suction pressure with the micrometer to reach a regime in which membrane fluctuates. Then increase the pressure to clearly visualize a tongue in the tip with a significant projection length (a few microns).

NOTE: The lowest applied pressure $\left(P_{0}\right)$ which allows sucking up the smallest membrane projection length $\left(L_{0}\right)$ will define the reference point $\alpha_{0}$. (Figure 7-A) All points of the curve will be measured according to this reference. $\left(\Delta \mathrm{L}=\mathrm{L}-\mathrm{L}_{0}\right.$ and $\left.\Delta \mathrm{P}=\mathrm{P}-\mathrm{P}_{0}\right)$

4.3.5. Increase the suction pressure with the micrometer in a stepwise manner until reaching $0.5-0.8 \mathrm{mN} / \mathrm{m}$. At each step, wait $5 \mathrm{sec}$ and take a snapshot of the tongue. This procedure at low tension regime enables to determine the bending modulus.

4.3.6. Keep on increasing the suction pressure from $0.5 \mathrm{mN} / \mathrm{m}$ to the rupture tension by adjusting the height of the water filled sliding on the rail (ranging from -2 to $-50 \mathrm{~cm}$ ) (Figure 7-B to 7-D). From this experiment at high tension regime, the area compressibility modulus, lysis tension and lysis strain will be measured.

4.3.7. Stretch About $15-20$ vesicles to acquire significant statistics. Each micropipette aspiration experiment takes between 7 and 10 minutes. Images analysis is performed using Leica LASAF software for measuring the projection length of the tongue, the diameter of vesicles and the radius of the capillary.

4.4 Measuring bending modulus, area compressibility modulus, lysis tension and lysis strain: To access these parameters, use the formalism established by E. Evans ${ }^{12}$. Calculate the suction pressure applied over the membrane from equation 1 :

$$
\Delta \mathrm{P}=\rho_{\mathrm{w}} \mathrm{g}(\mathrm{h}-\mathrm{h} 0)
$$

where $\mathrm{g}$ is the gravitational acceleration $\left(9.8 \mathrm{~m} \cdot \mathrm{s}^{-2}\right), \rho_{\mathrm{w}}$ is the density water $\left(\rho=1 \mathrm{~g} \cdot \mathrm{cm}^{-3}\right), \mathrm{h}$ is the position of the water tank and $h_{0}$ is the initial position where pressure is equal to zero.

Then calculate the membrane tension from the Laplace equation: 


$$
\sigma=\frac{\Delta P}{2} \frac{R_{P}}{1-\frac{R_{P}}{R_{V}}}
$$

where $\Delta P$ is the suction pressure on the micropipette, $R_{p}$ and $R_{v}$ are the micropipette and vesicle radii (outside the micropipette) respectively. The surface area strain $(\alpha)$ of the membrane is defined as:

$$
\alpha=\frac{A-A_{0}}{A_{0}}
$$

$A_{0}$ being the membrane area of the vesicle at the lower suction pressure. Calculate $\alpha$ from the increase in projection length $\Delta \mathrm{L}$ of vesicle inside the capillary tip according to Equation $4^{12}$ :

$$
\alpha=\frac{1}{2}\left(\frac{R_{P}}{R_{V}}\right)^{2}\left(1-\frac{R_{P}}{R_{V}}\right) \frac{\Delta L}{R_{P}}
$$

Under very low tension regime, smoothing of thermal bending undulations dominates the apparent expansion. Plot $\ln (\sigma)$ vs $\alpha$. At low- $\sigma$ values (typically $0.001-0.5 \mathrm{mN} \cdot \mathrm{m}^{-1}{ }^{13}$ ), this gives a straight line whose slope is linked to the bending modulus, $K_{b}$ (first term of the equation 5$)^{14}$ :

$$
\alpha=\frac{k T}{8 \pi K_{b}} \ln \left(1+\frac{A_{0} \sigma}{24 \pi K_{b}}\right)+\frac{\sigma}{K_{a}}
$$

Under high tensions $\left(>0.5 \mathrm{mN} \cdot \mathrm{m}^{-1}\right.$ ), membrane undulations are completely suppressed and membrane area increases as the result of increased spacing between molecules. In this regime, the second term of equation 5 dominates and give access to area compressibility modulus $\mathrm{K}_{\mathrm{a}}$ (Figure 8 and 9).

\section{REPRESENTATIVE RESULTS:}

With the protocol aforementioned, we have studied different synthetic Giant Unilamellar Vesicle, obtained from a phospholipid: 2-oleoyl-1-palmitoyl-sn-glycero-3-phosphocholine (POPC), a triblock copolymer: Poly(ethyleneoxide)- $b$-Poly(dimethylsiloxane)- $b$ Poly(ethyleneoxide) $\left(\mathrm{PEO}_{12}-b-\mathrm{PDMS}_{43}-b-\mathrm{PEO}_{12}\right)$ synthesised in a previous study ${ }^{13}$, and a diblock copolymer Poly(dimethylsiloxane)-b-Poly(ethyleneoxide) $\left(\mathrm{PDMS}_{27}-b-\mathrm{PEO}_{17}\right)$. It has been previously shown by our group that the association of triblock copolymer $\mathrm{PEO}_{8}-b-\mathrm{PDMS}_{22}-b$ $\mathrm{PEO}_{8}$ with phospholipid POPC leads to a huge decrease of membrane toughness of the resulting GHUVs (Giant Hybrid Unilamellar Vesicles) ${ }^{15}$. The measurement has been repeated for this study and extended to GUVs obtained from diblock copolymer and GHUV obtained from the association of this diblock copolymer and POPC.

The results are illustrated in figure 10 and table 1 . The area compressibility modulus and lysis strain for POPC are in perfect agreement with literature ${ }^{16}$. The measurement of the bending moduli of the hybrid vesicle has not yet been performed in the laboratory. Typical values are given for the polymersomes obtained. It is worth to mention that the toughness of membrane $\left(E_{c}\right)$ obtained from diblock copolymers is far beyond those obtained with triblock copolymer. More interestingly, with diblock copolymer it is possible to obtain Giant Hybrid Unilamellar 
Lipid/Polymer vesicles which present higher toughness than the liposomes, which is the main interest of such association.

FIGURES AND TABLE:

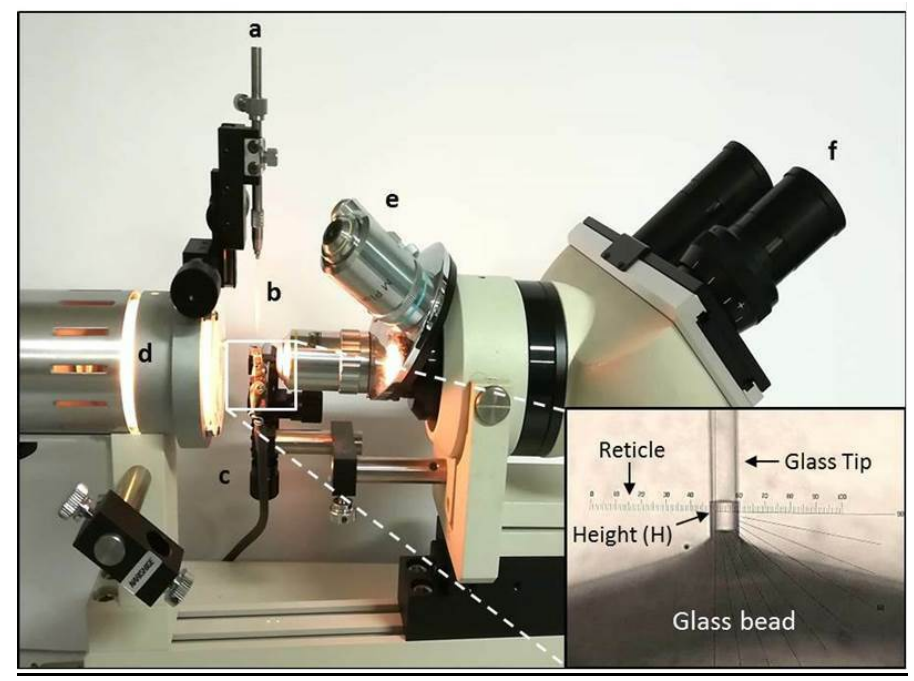

Figure 1: Microforge for polishing pipette tips. The picture shows the different parts of the device: metal pipette holder (a), glass capillary (b), micromanipulator heater zone (c), light source (d), 10x, 32x, 40x objectives (e), microscope oculars (f). In the insert, the pipette tip, immersed in a glass bead, is observed through a $32 x$ objective with reticle. The level of the molten glass into the tip has been fixed at an intermediate height $(\mathrm{H})$ after cooling. Pulling the tip away causes a sharp break.

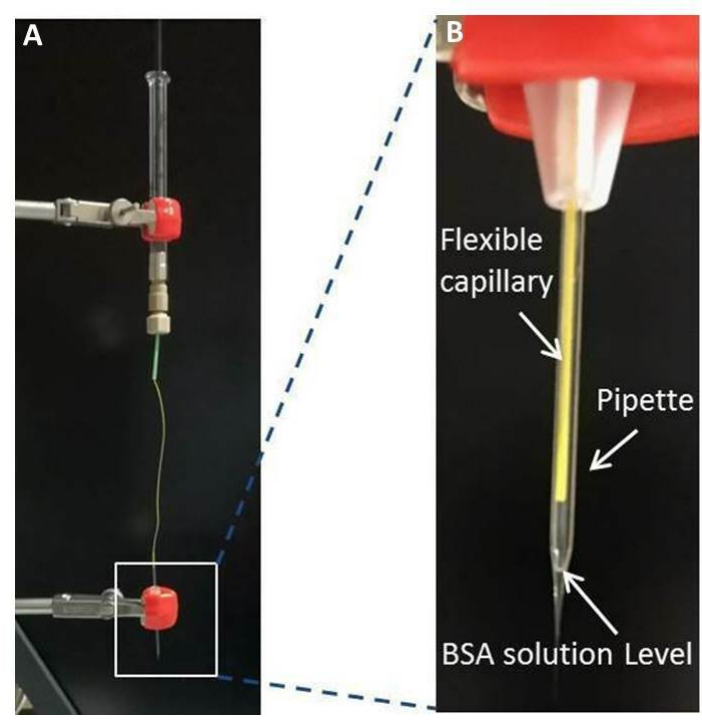

Figure 2: Experimental set up for coating and filling pipette tips. (A) A $500 \mu$ l glass syringe equipped with a flexible silica capillary is mounted to fill the micropipette with a glucose solution. (B) Magnification of the capillary lower part. During overnight immersion, the BSA solution level rises by capillarity up to $1 \mathrm{~cm}$ in length. The pipette is then filled with glucose by inserting a flexible capillary to remove unadsorbed BSA. 


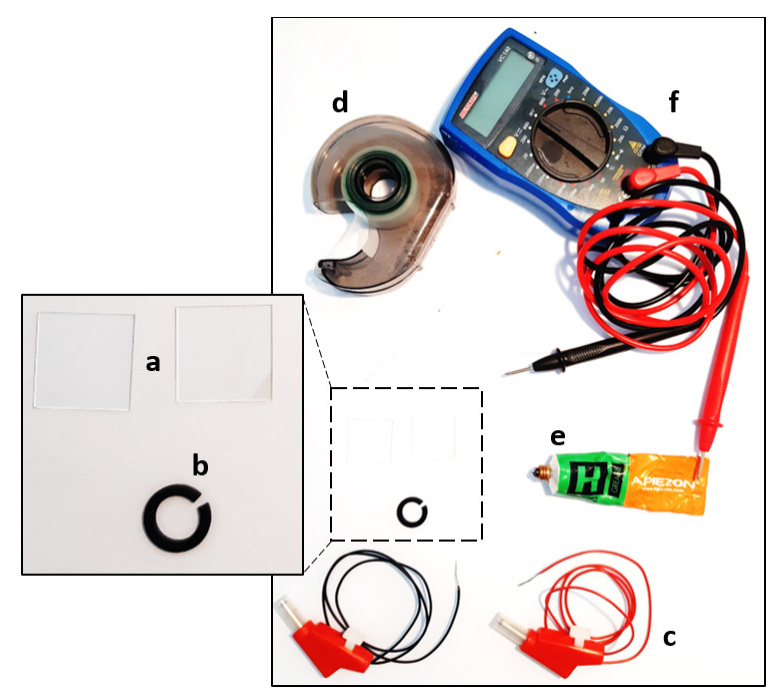

Figure 3: Materials to build an ITO based electroformation device. The device is composed of two glass slides coated on one side with Indium Tin Oxide (a). A rubber O-ring has been cut on one side to allow loading of solution inside the chamber and harvest of the GUVs suspension (b). The rubber O-ring is used as a spacer to separate the two slides. The slides are connected to the voltage generator via electric wires (c) and attached to the surface by adhesive tape (d). Silicon-free grease is used to seal the spacer with the slides (e). Ohmmeter is used to identify the ITO coated sides and check the conductivity (f).
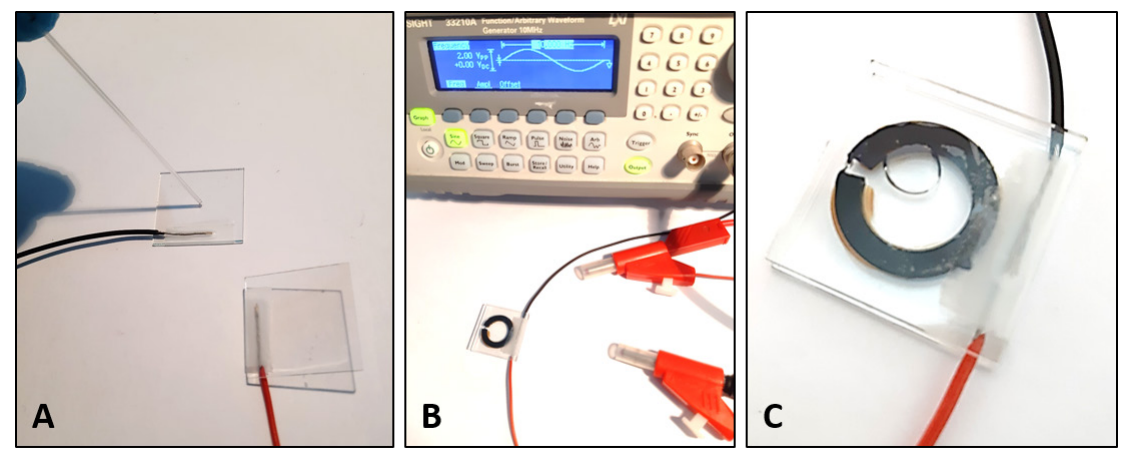

Figure 4: Electroformation set up. (A) Amphiphile solution is deposited using a glass capillary on the ITO coated sides of the glass plates. (B) After the assembly and the drying step, the chamber is connected to the voltage generator $(10 \mathrm{~Hz}, 2 \mathrm{~V})$ then filled with sucrose solution. (C) After electroformation, an air bubble is created inside the chamber to help the GUVs to detach from the surface. 


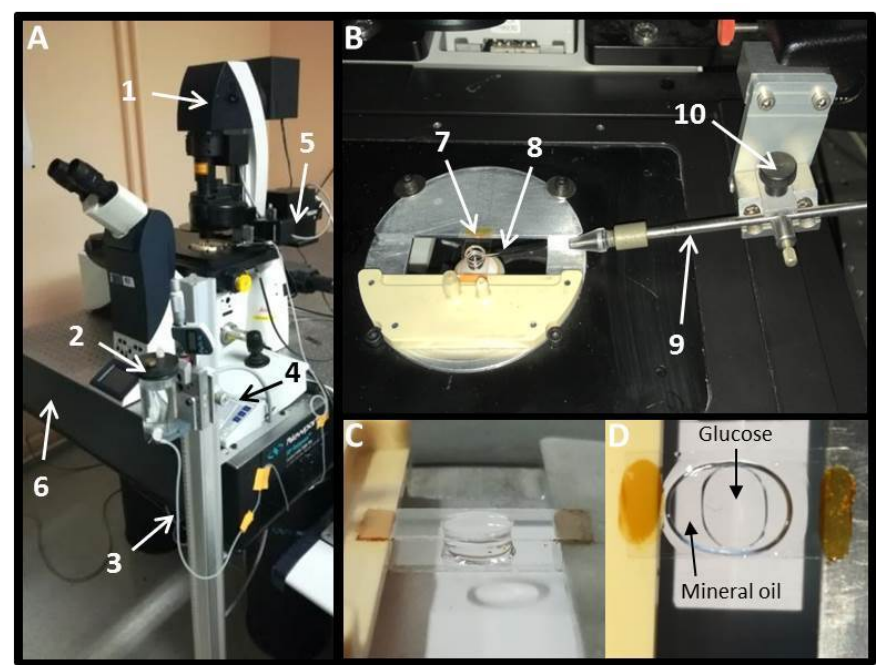

Figure 5: Micropipette Aspiration Set Up. (A) Components description: Fluorescence microscope (1), water filled tank and micrometer sliding on an Aluminum Optical Rail (2), silicone tubing conducting water flow from the reservoir to the micropipette (3), micromanipulator control panel (4), motor unit of the micromanipulator which allows $\mathrm{X}, \mathrm{Y}$ and $\mathrm{Z}$ displacement (5), vibration isolation table (6). (B) Magnification showing the homemade aluminum stage equipped with two glass slides (7), pipette (8) and pipette holder (9) mounted on the motor unit of the micromanipulator and fixed by clamping knob (10). Note that the pipette tip is immersed at the center of glucose meniscus. (C) Glass slides glued with vacuum grease on each side allowing the formation of glucose meniscus (Side view). (D) Glucose meniscus surrounded by mineral oil to prevent water evaporation (Top view).

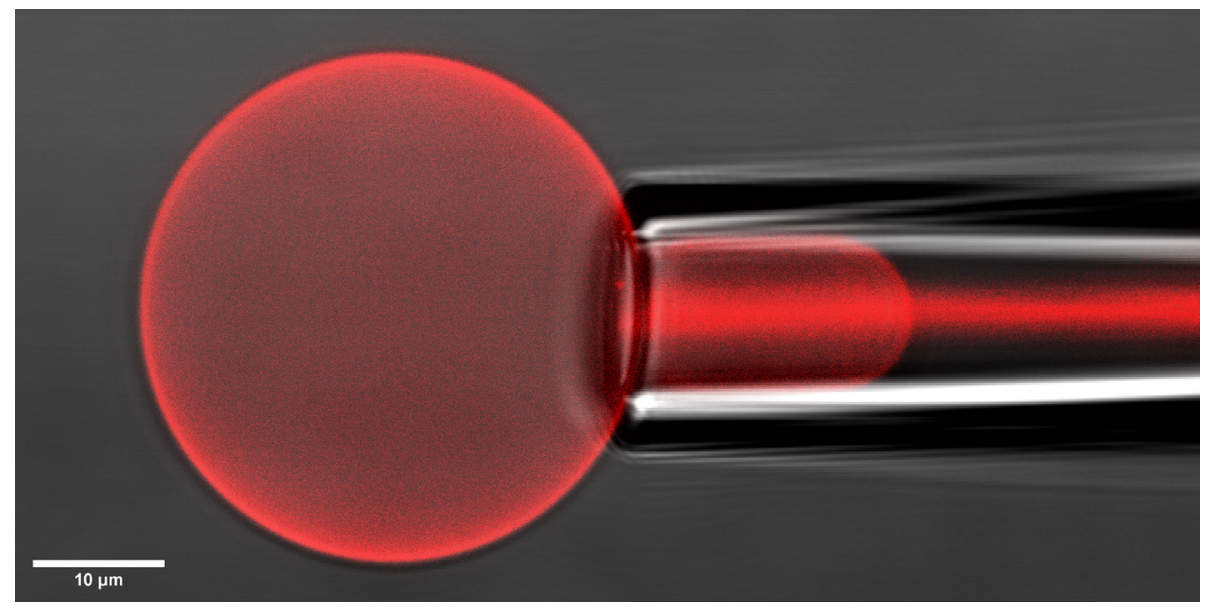

Figure 6: Image of a GUV under tension using micropipette aspiration. The red channel collecting the fluorescence from the rhodamine tagged lipid and the transmission channel (DIC) have been merged. 


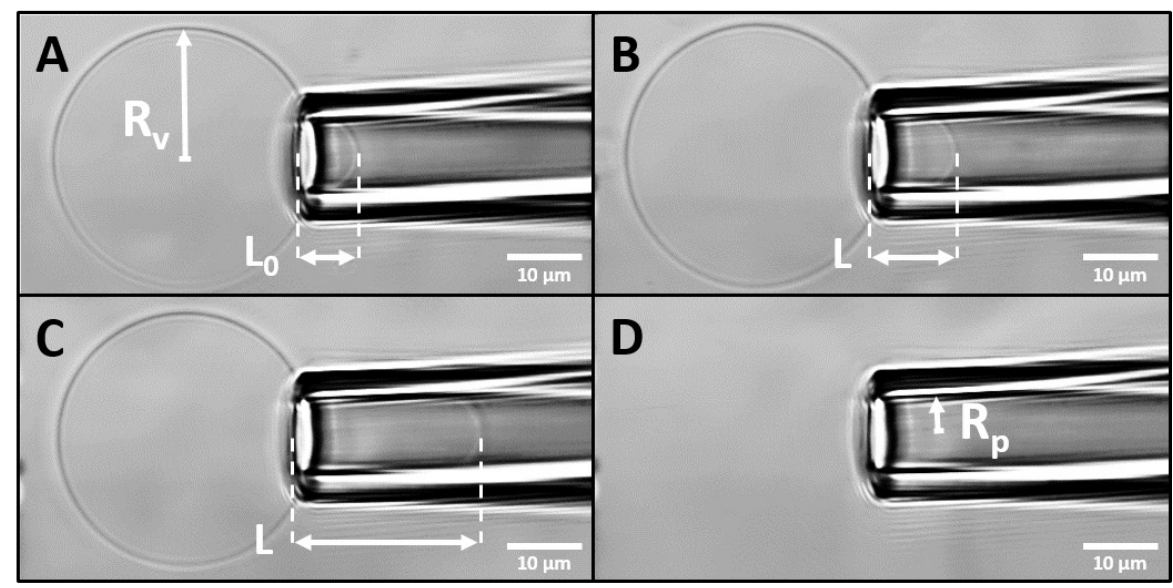

Figure 7: Images of a GUV at different suction pressure. (A) The lowest applied tension that induces a tongue formation is used as reference to determine the initial tongue length $\left(L_{0}\right)$ and the vesicle radius $\left(R_{v}\right)$. (B) Intermediate applied tension value with the associated tongue length (L). (C) Very high applied tension. (D) Image just after the membrane rupture where the pipette radius is measured $\left(R_{p}\right)$.

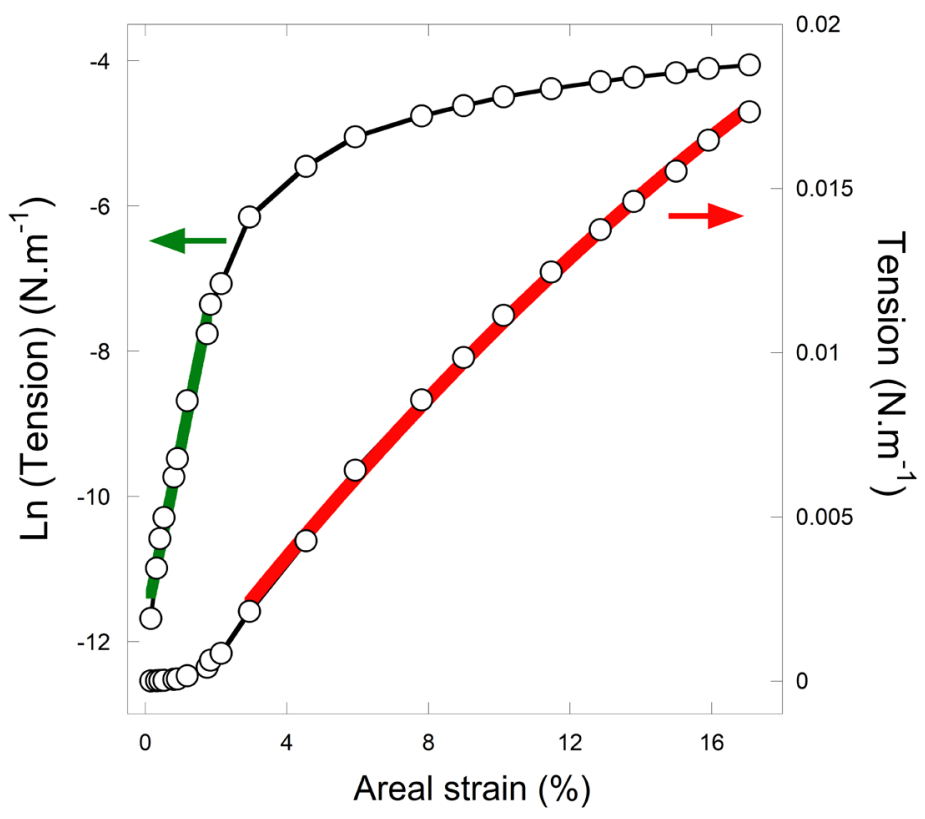

Figure 8: Representative stress-strain plot of GUVs obtained by micropipette aspiration. The same data set has been used to plot $\ln (\sigma)=f(\alpha)$ and $\sigma=f(\alpha)$. In the low tension regime $\ln (\sigma)$ vary linearly with $\alpha$ (green linear fit) and give access to the Bending Modulus $\left(\mathrm{K}_{\mathrm{c}}\right)$ whereas in the high tension regime $\sigma$ vary linearly with $\alpha$ (red linear fit) and give access to the Area Compressibility Modulus $\left(\mathrm{K}_{\mathrm{a}}\right)$. 


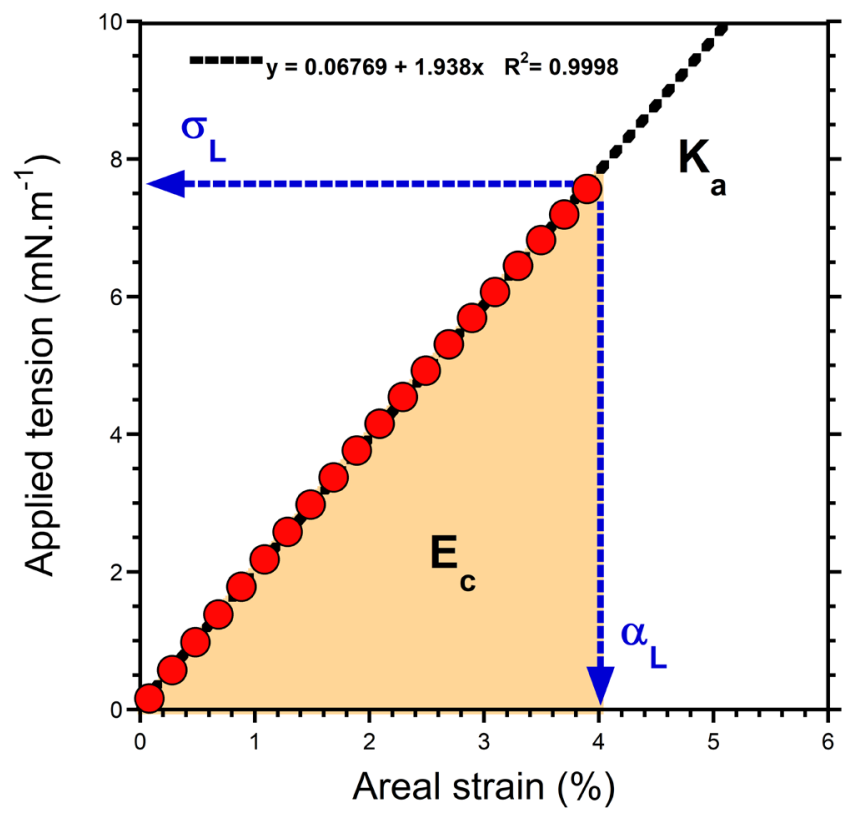

Figure 9: Representative stress-strain plot of GUV obtained by micropipette aspiration in the stretching regime. From the experimental curve several mechanical parameters of the GUVs can be determined. The Area Compressibility Modulus $\left(\mathrm{K}_{\mathrm{a}}\right)$ corresponds to the initial slope and is measured by fitting linearly the curve. The last measured point gives the Lysis Strain $\left(\alpha_{L}\right)$ and the Lysis Stress $\left(\sigma_{L}\right)$ values. Finally, the Cohesive Density Energy $\left(E_{c}\right)$ can be estimated by integrating the area under the curve (orange area).

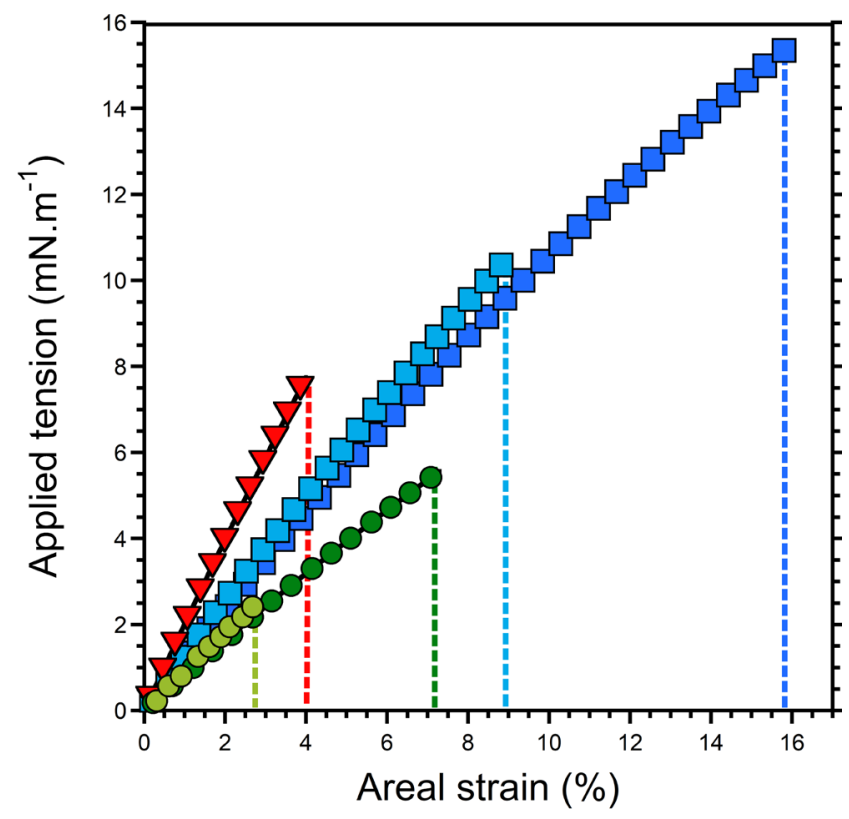

Figure 10: Representative stress-strain plots obtained for Liposome, Polymersome and Hybrid Polymer/Lipid vesicle. GUVs composed of 2-oleoyl-1-palmitoyl-sn-glycero-3-phosphocholine (POPC, red triangles), triblock copolymer (green circles), diblock copolymer (blue squares), triblock-based hybrid (light green circles) and diblock-based hybrid (light blue squares). The curves were obtained by averaging the measurements on at least 15 GUVs for each system. 


\begin{tabular}{|c|c|c|c|c|c|}
\hline & Ka & $\alpha_{L}$ & $\sigma_{\mathrm{L}}$ & $E_{c}$ & $\mathrm{~Kb}$ \\
\hline & $\left(\mathrm{mN} \cdot \mathrm{m}^{-1}\right)$ & $(\%)$ & $\left(\mathrm{mN} \cdot \mathrm{m}^{-1}\right)$ & $\left(\mathrm{mN} \cdot \mathrm{m}^{-1}\right)$ & $(\mathrm{kT})$ \\
\hline POPC & $194 \pm 15$ & $4 \pm 1$ & $8 \pm 2$ & $0.17 \pm 0.09$ & $21.1 \pm 0.4$ \\
\hline PDMS $_{27}-b-$ PEO $_{17}$ & $121 \pm 8$ & $16 \pm 4$ & $15 \pm 3$ & $1.37 \pm 0.67$ & $10.6 \pm 3.5$ \\
\hline PDMS $_{27}-b-$ PEO $_{17}$ & \multirow[t]{2}{*}{$132 \pm 13$} & \multirow[t]{2}{*}{$9 \pm 4$} & \multirow[t]{2}{*}{$10 \pm 3$} & \multirow[t]{2}{*}{$0.50 \pm 0.38$} & \multirow[t]{2}{*}{ - } \\
\hline + 5 wt. $\%$ POPC & & & & & \\
\hline $\begin{array}{l}\mathrm{PEO}_{12^{-}} b-\mathrm{PDMS}_{43^{-}}-b- \\
\mathrm{PEO}_{12}\end{array}$ & $84 \pm 13$ & $7 \pm 1$ & $6 \pm 2$ & $0.50 \pm 0.38$ & - \\
\hline $\begin{array}{l}\text { PEO }_{12}-b-\text { PDMS }_{43}-b- \\
\text { PEO }_{12}\end{array}$ & \multirow[t]{2}{*}{$91 \pm 11$} & \multirow[t]{2}{*}{$3 \pm 1$} & \multirow[t]{2}{*}{$2 \pm 1$} & \multirow[t]{2}{*}{$0.03 \pm 0.01$} & \multirow[t]{2}{*}{-} \\
\hline + 5 wt. $\%$ POPC & & & & & \\
\hline
\end{tabular}

Table 1: Mechanical parameters determined using micropipette aspiration techniques on GUVs composed of phospholipid, copolymers or both.

\section{DISCUSSION:}

The coating of the micropipette is one of the key points to obtain reliable measurements. Adhesion of the vesicle to the micropipette has to be prevented, and a coating is commonly used in literature ${ }^{17-21}$, with BSA, $\beta$-casein or surfasil. Details of the coating procedure are rarely mentioned.

Dissolution of the BSA should be performed for at least 4 hours under agitation in order to achieve good solubilization. Nevertheless, the filtration step is still required to remove any aggregates that may obstruct the micropipette tip. If BSA is not well dissolved, most of it will be removed by filtration, and coating will be ineffective. The ideal concentration and dissolution time are respectively $0.8-1 \mathrm{wt} . \%$ and $4 \mathrm{~h}$.

Another critical point is to insure a constant osmotic pressure inside and outside vesicle during measurement. An increase of glucose concentration due to water evaporation during the experiment can lead to deflation of the vesicle and perturb the measurement (underestimation of $\mathrm{K}_{\mathrm{a}}$, etc). The deposition of an oil layer is mandatory to prevent this phenomenon. (Figure 3-D) To check the efficiency of the oil layer, a constant aspiration pressure of few $\mathrm{mN}^{-1} \mathrm{~m}^{-1}$ can be applied on a vesicle during $5 \mathrm{mn}$, and the length $\mathrm{L}$ of the tongue inside the capillary should be constant.

The last critical point is the pre-stress step (section 3.3), rarely mentioned in literature ${ }^{20}$. This step is necessary to remove buds, tubes and excess surface area of vesicle and get reproducible results from a vesicle to another.

The micropipette aspiration method can be applied on all GUVs, as long as they present a fluid membrane (e.g. $T_{\text {electroformation }}>T_{m}$ of lipids) and possess a bending modulus below $100 \mathrm{kT}$. In the case of thick and viscous membrane, even in the fluid state, two pipettes can be used to measure the moduli ${ }^{22}$. Micropipette aspiration technique presents the great advantage to give access to several parameters (Bending and area compressibility moduli) and this is the only 
technique available to directly access to area compressibility modulus of GUV's membrane. Although this technique is well known for a long time it is still commonly used by numerous scientific communities (biophysicists, physico-chemists, chemists...). The micropipette aspiration method will continue to be considered as a significant technique in the future, especially to investigate further membrane properties of advanced synthetic cell (e.g. Hybrid Polymer/Lipid Vesicles and protocells).

\section{ACKNOWLEDGMENTS:}

The authors gratefully acknowledge the ANR for financial support (ANR Sysa).

\section{DISCLOSURES:}

The authors have nothing to disclose.

\section{REFERENCES:}

1 Bangham, A. D., Standish, M. M. \& Watkins, J. C. Diffusion of univalent ions across the lamellae of swollen phospholipids. Journal of Molecular Biology. 13 (1), 238-IN227, (1965).

2 Discher, D. E. \& Eisenberg, A. Polymer vesicles. Science. 297 (5583), 967-973, (2002).

3 Hammer, D. et al. Polymersomes: vesicles from block copolymers. Annals of Biomedical Engineering. 28 (SUPPL. 1), (2000).

4 Le Meins, J. F., Schatz, C., Lecommandoux, S. \& Sandre, O. Hybrid polymer/lipid vesicles: state of the art and future perspectives. Materials Today. 16 (10), 397-402, (2013).

5 Schulz, M. \& Binder, W. H. Mixed Hybrid Lipid/Polymer Vesicles as a Novel Membrane Platform. Macromolecular Rapid Communications. 36 2031-2041, (2015).

6 Schneider, M. B., Jenkins, J. T. \& Webb, W. W. Thermal fluctuations of large quasispherical bimolecular phospholipid vesicles. J. Phys. France. 45 (9), 1457-1472, (1984).

7 Dimova, R. Recent developments in the field of bending rigidity measurements on membranes. Adv Colloid Interface Sci. 208 225-234, (2014).

8 Rodríguez-García, R. et al. Polymersomes: smart vesicles of tunable rigidity and permeability. Soft Matter. 7 (4), 1532-1542, (2011).

9 Angelova, M. I. \& Dimitrov, D. S. Liposome electroformation. Faraday Discussions of the Chemical Society. 81 303-311, (1986).

10 Dao, T. P. T. et al. Membrane properties of giant polymer and lipid vesicles obtained by electroformation and pva gel-assisted hydration methods. Colloids and Surfaces A: Physicochemical and Engineering Aspects. 533 347-353, (2017).

11 Pereno, V. et al. Electroformation of Giant Unilamellar Vesicles on Stainless Steel Electrodes. ACS omega. 2 (3), 994-1002, (2017).

12 Evans, E. \& Rawicz, W. Entropy-driven tension and bending elasticity in condensed-fluid membranes. Phys Rev Lett. 64 (17), 2094-2097, (1990).

13 Dao, T. P. T. et al. Modulation of phase separation at the micron scale and nanoscale in giant polymer/lipid hybrid unilamellar vesicles (GHUVs). Soft Matter. 13 (3), 627-637, (2017).

14 Helfrich, W. - Elastic properties of lipid bilayers: theory and possible experiments. - Z Naturforsch C. 1973 Nov-Dec;28(11):693-703. (- 0341-0471 (Print)), T - ppublish, (1973). 
15 Dao, T. P. T. et al. The combination of block copolymers and phospholipids to form giant hybrid unilamellar vesicles (GHUVs) does not systematically lead to "intermediate" membrane properties. Soft Matter. 14 (31), 6476-6484, (2018).

16 Shoemaker, S. D. \& Kyle Vanderlick, T. Material Studies of Lipid Vesicles in the La and La-Gel Coexistence Regimes. Biophysical Journal. 84 (2), 998-1009, (2003).

17 Longo, M. L. \& Ly, H. V. in Methods in Membrane Lipids (ed Alex M. Dopico) 421-437 (Humana Press, 2007).

18 Chen, D. \& Santore, M. M. Hybrid copolymer-phospholipid vesicles: phase separation resembling mixed phospholipid lamellae, but with mechanical stability and control. Soft Matter. 11 (13), 2617-2626, (2015).

19 Mabrouk, E. et al. Formation and material properties of giant liquid crystal polymersomes. Soft Matter. 5 1870-1878, (2009).

20 Henriksen, J. et al. Universal behavior of membranes with sterols. Biophys J. 90 (5), 1639-1649, (2006).

21 Ly, H. V., Block, D. E. \& Longo, M. L. Interfacial Tension Effect of Ethanol on Lipid Bilayer Rigidity, Stability, and Area/Molecule: A Micropipet Aspiration Approach. Langmuir. 18 (23), 8988-8995, (2002).

22 Bermudez, H., Hammer, D. A. \& Discher, D. E. Effect of Bilayer Thickness on Membrane Bending Rigidity. Langmuir. 20 540-543, (2004). 